\title{
Komplikationsmanagement nach Eingriffen an der Wirbelsäule
}

\author{
Jan Baranczyk, Hans Peter Langen, Ina Schmidt, Jörg Schmidt
}

\section{Zusammenfassung}

Für das Auftreten von Komplikationen sind in der Regel die Konstitution des Patienten mit all seinen physiologischen und auch pathophysiologischen Faktoren verantwortlich, aber auch der Operateur selbst mit all den Möglichkeiten der indikatorischen und operativen Fehlleistungen, die letztendlich auch im weiteren Verlauf zu Problemen führen können, die einen normalen und gradlinigen Ausgang der Behandlung verhindern. Nur eine ausreichende Kenntnis der Möglichkeiten, eine Komplikation zu erzeugen, macht den Behandler im Verlauf der Therapie aufmerksam, nach den Komplikationen zu suchen und sie so schnell wie möglich zu beheben. Im Folgenden sollen die wesentlichen Möglichkeiten von Fehlleistungen im Behandlungsverlauf dargestellt werden. Lösungsansätze werden vorgeschlagen.

\section{Management of Complications after Spine Surgery}

The patient's constitution with all his/ her physiological and pathophysiological factors are mostly responsible for complications; however, also the surgeon's indication and operating errors may be responsible for problems occurring in the course of rehabilitation. Only an adequate knowledge of how possible complications may occur can enable the therapist to detect complications during the treatment and solve them as quickly as possible. The present article aims to demonstrate the main errors made during medical treatment and rehabilitation and proposes appropriate solutions.

\section{Vorwort}

„Quae nocent, docent“

„Die gebräuchlichen chirurgischen Operationslehren beschränken sich darauf, den normalen Gang einer Operation in Wort und Bild darzustellen, sie weisen dabei vielleicht auch auf diese oder jene Gefahr hin und schildern technische Schwierigkeiten, aber sie machen es sich nicht zur Aufgabe, all die Klippen zu veranschaulichen, an denen der chirurgische Anfänger und auch mancher Fortgeschrittene schon Schiffbruch erlitten hat, sie weisen nicht auf jeder Seite eindringlich darauf hin, dass nur ein kleiner

OP-JOURNAL 2009; 25: 42-50

(c) Georg Thieme Verlag KG Stuttgart · New York DOI 10.1055/s-0029-1185492

Zahn in dem großen Räderwerk der Maschine, die mit jeder Operation in Gang gesetzt wird, defekt zu sein braucht, um das Gelingen des ganzen Eingriffs infrage zu stellen. Nur wer die Fehler, die bei einer Operation zu vermeiden sind, möglichst restlos kennt, wird ihnen entgehen. Die blasse Angst allein, die das Hirn blutleer macht und die Knie schlottern lässt, ist keine gute Beraterin; sie drängt zu Verzweiflungsentschlüssen, und diese sind meist nicht gut" [30].

Diesen bereits 1923 geschriebenen Worten von Stich und Makkas in dem Buch „Fehler und Gefahren bei chirurgischen Eingriffen" fehlt heute, fast 100 Jahre später, nichts an Aktualität. Die Eingriffe auf den Gebieten der Unfallchirurgie und Orthopädie werden immer komplexer, technisch aufwendiger und komplikationsträchtiger.
Die Möglichkeiten, gerade auf dem Gebiet der Wirbelsäulenchirurgie, sind noch lange nicht ausgereizt. Bewährte Techniken, z.B. auf dem Gebiet der Osteosynthese an der Wirbelsäule, werden durch die moderne 3-dimensionale Bildgebung in Verbindung mit der Navigation technisch aufwendiger; sie bergen bei allen Vorteilen für die Sicherheit unserer Patienten bei routinierter Anwendung aber erheblich mehr Möglichkeiten der Fehlleistungen. Neue Verfahren, wie z.B. die Vertebroplastie und Kyphoplastie, finden zu rasch eine weite Verbreitung und können überreizt werden. Hier werden im Laufe der Zeit noch Probleme des Komplikationsmanagements auf uns zukommen, die wir derzeit noch gar nicht überblicken können.

Oftmals liegen die Probleme im Kleinen. Sind alle Instrumente auf den Sieben und auch steril? Sind alle benötigten Implantate auch vom Einkauf bestellt und geliefert? Habe ich mich ausreichend mit dem Patienten befasst und die zu versorgende Verletzung auf ihre Eigenheiten reduziert? Bin ich in meiner heutigen Verfassung eigentlich der richtige Operateur?

Probleme entstehen bei der Indikationsstellung, dem operativen Vorgehen und auch in der Nachbehandlungsphase. Überall hier müssen wir Fehlleistungen erkennen, eingestehen und beheben, also ein Komplikationsmanagement betreiben.

Wer ein operatives Verfahren anwendet, muss die wesentlichen Komplikationen kennen und sich sicher sein, sie auch zu beherrschen.

\section{Komplikationsmanagement nach Fehlindikationen}

Voraussetzung für eine regelrechte Behandlung der Wirbelsäulenfrakturen und den komplikationsfreien Verlauf der Behandlung ist zunächst die gründliche 


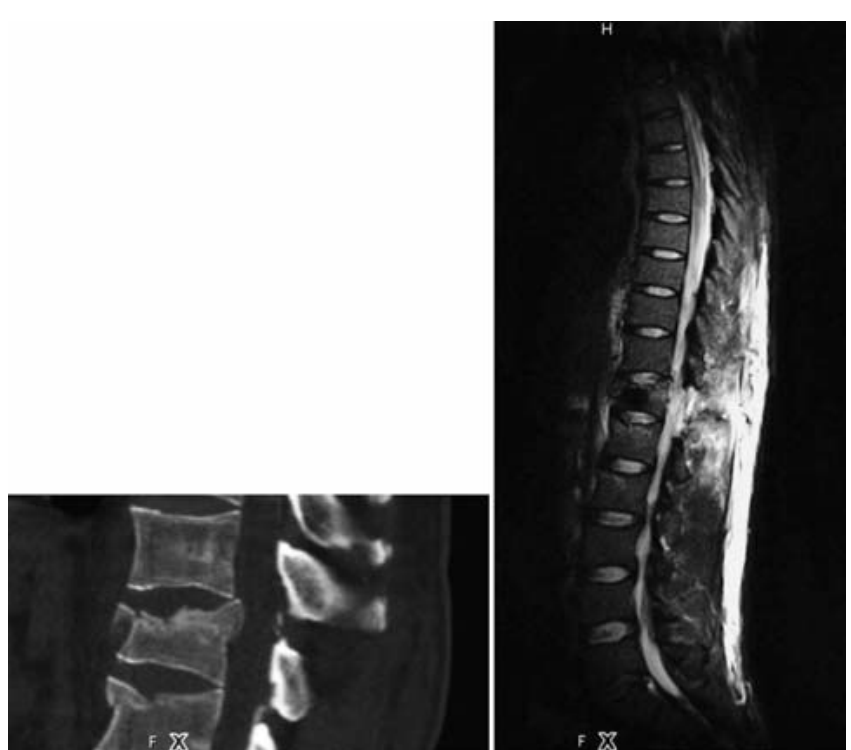

Abb. 1 BWK-12 und LWK-1-Fraktur im $\mathrm{CT}$. Die gleiche Fraktur im MRT zeigt Verletzungen der dorsalen Bänder, also eine instabile B-Fraktur.
Diagnostik und Klassifizierung der Verletzung. Hierzu gehört neben Anamneseerhebung und der körperlichen sowie neurologischen Untersuchung die Röntgen- und CT-Diagnostik der Wirbelsäule $[2,3,6,7,19,23,35]$. Die Klassifikation der Verletzung erfolgt nach der Einteilung der Arbeitsgemeinschaft für Osteosynthesefragen (AO). Anhand der Klassifikation wird die Art der Behandlung festgelegt.

Eine konservative Behandlung führt nur bei exakter Indikationsstellung zum gewünschten Erfolg. Stabile Frakturen des Typs A1 und A2 können in der Regel konservativ behandelt werden. Eine Ausnahme sind die sog. Kneifzangenbrüche (auch Pinzer-Fraktur) (A2.3), die aufgrund der Zerreißung beider benachbarter Bandscheiben dorsoventral bisegmental versorgt werden sollten. Bei geplanter konservativer Behandlung einer A-Verletzung muss immer ein zusätzlich zum CT durchgeführtes MRT zum Ausschluss diskoligamentärer Instabilitäten durchgeführt werden. Liegt eine diskoligamentäre Instabilität vor, ist die Fraktur in der Klassifikation als B-Fraktur anzusehen und damit nicht mehr stabil, eine operative Versorgung ist erforderlich (Abb. 1).

Vor allem bei A3.1-Verletzungen wird die alleinige ventrale operative Versorgung diskutiert. Als problematisch kann sich die intraoperative Lagerung erweisen. Wird der Patient zur besseren Übersicht über das OP-Gebiet in Seitneigung versorgt, kann eine skoliotische Verbiegung der Wirbelsäule mit entsprechenden langfristigen Haltungsproblemen fixiert werden. Auch die Rekonstruktion der Wirbelkörperhöhe und damit der physiologischen Lordosierung der Wirbelsäule ist ausgesprochen schwierig. Sollte sich der Operateur zum einzeitigen und alleine ventralen Vorgehen entschließen, kann sich der temporäre Einsatz des Fixateur externe von dorsal zur temporären Reposition der Wirbelsäule als hilfreich erweisen. Präoperativ muss auch in diesen Fällen eine MRT-Untersuchung das Vorliegen einer A-Verletzung beweisen.

Haupteinsatzgebiete der in den letzten Jahren zunehmend angewendeten Vertebro- und Kyphoplastie sind schmerzhafte osteoporotische Wirbelkörpersinterungen und Wirbelkörperosteolysen bei maligner Erkrankung [22]. Geeignet für die Kyphoplastie sind frische Sinterungen mit einer Höhenminderung von über einem Drittel bis zur halben Wirbelkörperhöhe. Mit Sorgfalt muss insbesondere bei degenerativ veränderten Wirbelsäulen die Lokalisation des schmerzverursachenden Wirbels erfolgen. Hier ist die MRT mit der Darstellung des Knochenmarködems in frisch gesinterten Wirbelkörpern zu fordern.

Die Indikation zur Vertebro- und Kyphoplastie ist derzeit im Wesentlichen auf die stabilen Frakturtypen A1.1-A1.3 begrenzt, vereinzelt wurde auch die Anwendung bei höhergradigen Verletzungen beschrieben. Hierbei steigt jedoch das Risiko von Komplikationen. Diese liegen vor allem in der Nichtbeachtung der Instabilitäten des diskoligamentären Komplexes mit verbleibenden Instabilitäten der Wirbelsäule; daraus resultieren
Schmerzen und neurologische Veränderungen, die mit einer Spondylolisthese vergleichbar sind. Die Folge hier sind aufwendige dorsoventrale Rekonstruktionen, bei denen mit sehr viel Aufwand der zementgefüllte Wirbelkörper entfernt werden muss.

Die Grundlage der Versorgung einer Verletzung ist es, die Verletzung auf ihre Eigenheiten zu reduzieren und zu klassifizieren. Danach kann eine klassifikationsgerechte Versorgung erfolgen.

Fehleinschätzungen bei der Klassifikation und der sich daraus ergebenden Indikation zur konservativen oder nicht operativen Behandlung haben also für den Patienten weitreichende Folgen $[1,4]$. Bei der Wahl der falschen OPMethode können dem Patienten unerwünschte Nachteile entstehen, die sich z.B. aus nicht ausreichender Stellungskorrektur oder Stabilisierung ergeben können [16]. Bei unterlassener operativer Behandlung können neurologische Symptome auftreten oder der Patient hat im Verlauf Beschwerden mit anhaltenden Schmerzen und Bewegungseinschränkungen bis hin zu psychischen Problemen aufgrund von Schmerzen, neurologischen Störungen oder der veränderten Körperhaltung [13].

\section{Komplikationsmanagement nach "operativen Fehlleistungen“}

Je nachdem, was als Komplikation erfasst wird, schwankt die Häufigkeit unerwünschter Ereignisse bei der Versorgung von Wirbelsäulenverletzungen zwischen 5 und 25\%. Meist handelt es sich um operationstechnische Fehlleistungen oder implantatbedingte Probleme.

Die intraoperativen Komplikationen bei der operativen Versorgung von Wirbelsäulenverletzungen resultieren im Wesentlichen aus der Nähe zu anatomischen Strukturen. Dies sind im Bereich der ganzen Wirbelsäule Nervenwurzeln und Myelon. Im Bereich der Halswirbelsäule sind sowohl die Besonderheiten des Übergangs von $\mathrm{CO}-\mathrm{C} 2 \mathrm{zu}$ beachten als auch die engen Lagebeziehungen zur intraossär verlaufenden Arteria vertebralis, den hirnzuführenden Gefäßen und Hirnnerven. An der Brustwirbelsäule und Lendenwirbelsäule sind bei Eingriffen die Aorta und Lungen sowie Bauchorgane wie die Milz gefährdet $[10,15]$.

In der aktuellen Literatur sind Blutungen und Verletzungen der Dura noch vor 


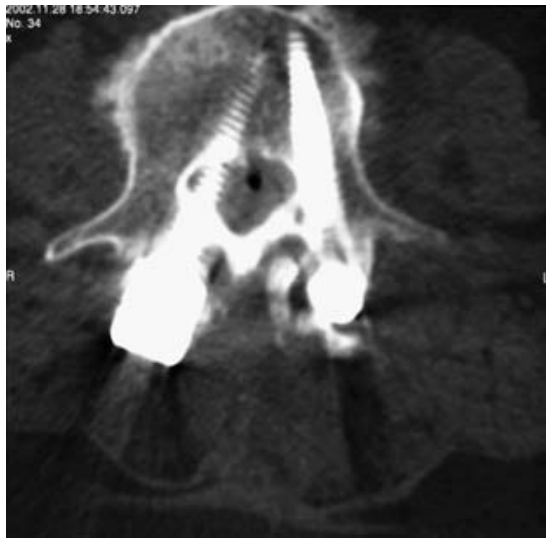

Abb. 2 Schraubenfehllage rechts durch den Spinalkanal nach dorsaler Instrumentierung.

Materialfehllagen die häufigsten Komplikationen [27].

\section{Dorsale Instrumentierung}

Die häufigsten Komplikationen bei der dorsalen Instrumentierung betreffen die Lage der Pedikelschrauben. Neben der Höhenlokalisation ist die genaue Einstellung der Pedikeleingangsebene mit dem Bildwandler während der Operation essenziell. Schraubenlängen und Durchmesser können ebenso wie der Einbringungswinkel orientierend anhand der präoperativen CT-Untersuchung bestimmt werden. Die Lage der Schrauben und Kontrolle der Reposition ist entweder intraoperativ durch einen 3-D-Scan oder postoperativ mit einer CT-Untersuchung zwingend zu überprüfen.

Bei einer zu weit medial verlaufenden Pedikelschraube ist die Perforation des Spinalkanals mit Verletzung der Dura oder des Myelons bzw. der Cauda equina möglich, aus der Lähmungen oder andere Nervenaffektionen resultieren können. Treten die Pedikelschrauben nach ventral aus dem Wirbelkörper heraus, besteht die Gefahr der Verletzung der Aorta oder der Iliakalgefäße, die auch noch im Verlauf auftreten kann (Abb. 2). Übertreten die Pedikelschrauben die kraniale oder kaudale Pedikelkortikalis, können Affektionen der Nervenwurzeln auftreten. Fehllagen der Schrauben müssen beim Auftreten von neurologischen Symptomen korrigiert werden. Fehlen neurologische Störungen, können die Schrauben, insbesondere beim lateralen Heraustreten oder nur geringer Tangierung des Spinalkanals ohne Duraaffektion belassen werden, wenn sie im Pedikel stabil liegen. Eine Fehllage der Pedikelschrauben im Zwischenwirbelraum kann ebenso wie der Ausbruch aus dem
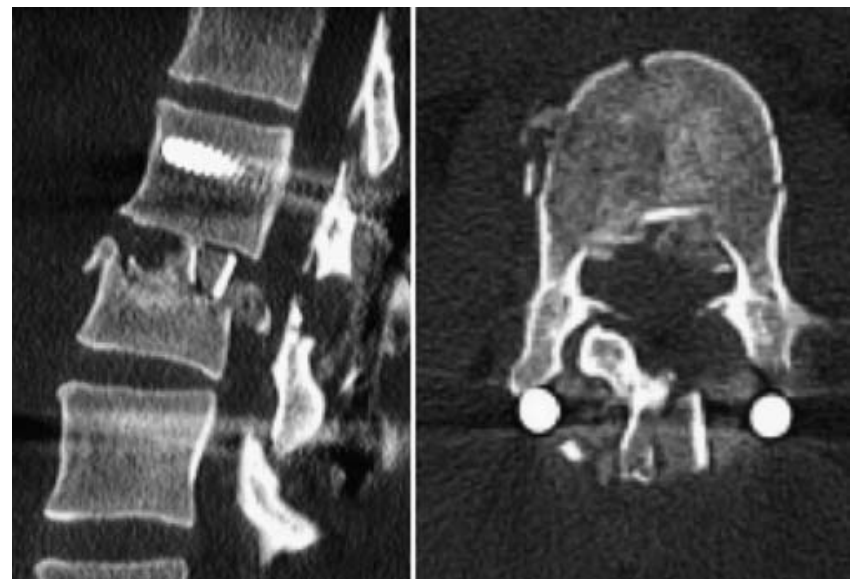

Abb. 3 Spandislokation in den Spinalkanal nach PLIF.

Pedikel in eine instabile Versorgung münden, hier müssen die Pedikelschrauben auf jeden Fall umgesetzt und ergänzt werden.

Eine weitere, seltene, intraoperative Fehlleistung stellt der iatrogene Bruch der Bogenwurzel dar, bei seinem Auftreten besteht die Möglichkeit des Umsetzens der Pedikelschrauben, auch die zusätzliche Zementierung wird beschrieben. Intraoperative Rippenverletzungen bedürfen in der Regel keiner spezifischen Behandlung.

Die Lage der Pedikelschrauben ist zu kontrollieren. Eine Revision erfolgt bei Instabilität oder neurologischen Komplikationen.

\section{Ventrale Fusion}

Die Komplexität der ventralen Versorgung bedingt ein höheres Ausmaß und einen höheren Schwierigkeitsgrad möglicher Komplikationen. Bei ventralen Stabilisierungen an der Brustwirbelsäule und oberen Lendenwirbelsäule sind transthorakale Zugänge notwendig. Hier hat die in den letzten Jahren vollzogene Veränderung des Zugangs vom offenen hin zum thorakoskopischen Vorgehen zu einer Reduktion der Zugangsmorbidität geführt. Dennoch besteht während des Eingriffs die Gefahr der Verletzung von wirbelsäulennahen Venengeflechten, der Lunge, der Aorta oder von intraabdominellen oder retroperitonealen Strukturen und Organen. Der transthorakale Zugang bedingt die Notwendigkeit einer qualifizierten anästhesistischen Betreuung (Doppellumentubus) und weiterer postoperativer Überwachung (Pleuradrainage). Als Komplikationen können u.a. ein persistierender Pneumo- thorax und rezidivierende Pleuraergüsse auftreten.

Diese bedürfen allerdings nur sehr selten weiterer chirurgischer Maßnahmen. Zugangsbedingt auftretende Rippenfrakturen können durch die Anwendung von Lokalanästhetika beim Abschluss der Operation in ihrer Schmerzhaftigkeit reduziert werden. Insgesamt wird der transthorakale Zugang in der Regel von den Patienten sehr gut toleriert.

Auch bei der ventralen Stabilisierung können Implantat- und Knochentransplantatfehllagen auftreten, die je nach entsprechendem Befund einer Korrektur bedürfen.

Bei der ventralen Spaneinbringung durch eine PLIF kann es zum unbemerkten Verbleib von Knochenspänen im Spinalkanal kommen. Trotz der dazu notwendigen Hemilaminektomie sind neurologische Störungen nicht ausgeschlossen. Eine Revision des Spinalkanals wird dann notwendig, falls eine komplette Laminektomie ausgeführt wird und muss zusätzlich ventral stabilisiert werden, um konsekutiven Instabilitäten mit Resorption des eingebrachten Spanes und damit einem Korrekturverlust vorzubeugen (Abb. 3).

Probleme bei der ventralen Fusion sind häufig durch die Zugangsmorbidität bestimmt. Der Operateur muss die Zugänge und ihre Komplikationen kennen und beherrschen.

\section{Osteosynthesen an der Halswirbelsäule}

Die Häufigkeit von Komplikationen bei Osteosynthesen an der Halswirbelsäule ist nicht höher als bei Operationen im 

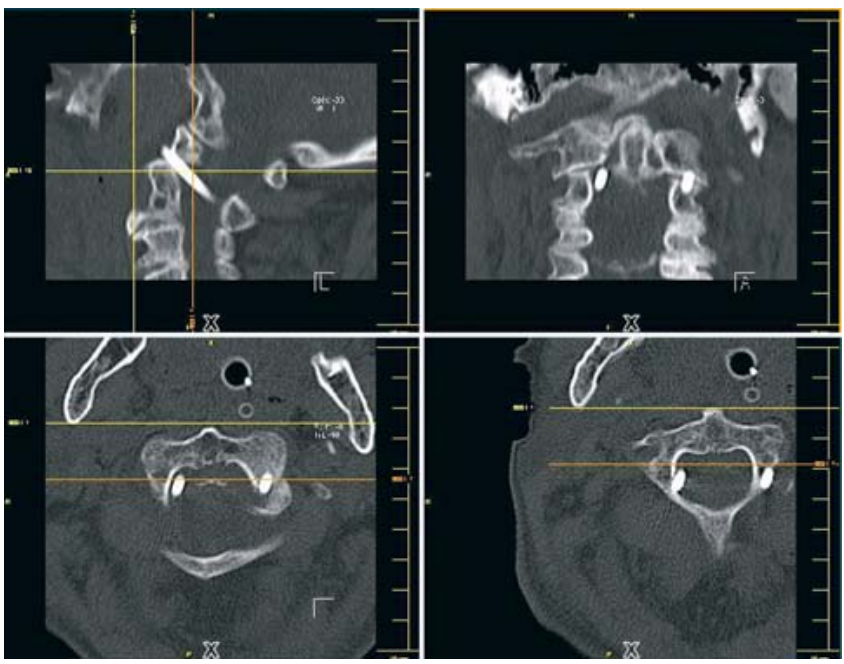

Abb. 4 Schraubenfehllage rechts nach transartikulärer Verschraubung $\mathrm{C} 2 / \mathrm{C} 1$ nach Magerl.

übrigen Wirbelsäulenbereich. Intraoperative Komplikationen wie schwer stillbare Blutungen, die Perforation eines großen Gefäßes, eine Wurzelläsion oder die Verletzung der Trachea oder des Ösophagus sind schwerwiegend, aber selten. Wie auch bei der Versorgung der thorakolumbalen Wirbelsäule sind Fehllagen von Osteosynthesematerial und eingebrachten Knochenspänen möglich [24]. Intraoperative Navigation und 3-dimensionale Bildgebung sind während der Operation hilfreich.

Die verschiedenen operativen Verfahren der Versorgung von Verletzungen der oberen Halswirbelsäule stellen hohe Anforderungen an die räumliche Orientierung des Operateurs (Abb.4).

Bei der dorsalen Instrumentierung an der Halswirbelsäule ist die Verankerung der Schrauben in den Massae laterales technisch einfacher, aber auch aufgrund der nur kurzen möglichen Schraubenlängen weniger stabil. Die transpedikuläre dorsale Instrumentierung an der Halswirbelsäule bietet mehr Stabilität, stellt jedoch aufgrund der engen Lagebeziehung zwischen Spinalkanal und Arteria vertebralis höchste Ansprüche an den Operateur. Bei der Verletzung der intraossär verlaufenden Arteria vertebralis kommt es zu Ischämiezeichen des Hirnstamms, die sich in postoperativer Übelkeit, Brechreiz, Dysphagie, Ohrensausen und ähnlichen unspezifischen Symptomen zeigen können.

Bei der ventralen Versorgung der unteren Halswirbelsäule durch eine Spondylodese besteht zugangsbedingt die Gefahr der Verletzung der Schilddrüse mit der Konsequenz kräftiger Blutungen, die insbesondere beim Vorliegen einer Struma erhöht ist. Eine (einseitige) Verletzung des N. recurrens resultiert in postoperativer Heiserkeit. Eine Verletzung der hirnversorgenden Gefäße bedarf einer operativen Wiederherstellung.

Die Anwendung eines Halo-Fixateurs setzt eine intakte Struktur der Schädelkalotte voraus. Beim Einbringen der Kopfstifte darf die Verankerung nur in der Lamina externa des Schädelknochens erfolgen [25]. Werden die ventralen Stifte zu tief platziert, besteht die Gefahr der Perforation der Orbita. Mögliche Gefäß- und Nervenläsionen betreffen die N. supraorbitalis und supratrochlearis bei zu weit ventral eingebrachten Stiften und Äste des N. auriculotemporalis und der A. temporalis bei zu weit dorsal eingebrachten Stiften. Beim Auftreten von Sensibilitätsstörungen werden die Schrauben neu platziert.

\section{Vertebro- und Kyphoplastie}

Bei indikationsgerechtem Einsatz ist die Komplikationsrate für die Vertebro- und Kyphoplastie relativ gering. Für die Platzierung des Instrumentariums gelten die gleichen Anforderungen wie für die dorsale Instrumentierung. Entsprechend können beim Abweichen der Kanüle Strukturen des Spinalkanals und Rückenmarks bzw. der Cauda equina und in Abhängigkeit von der Höhe der Versorgungen Verletzungen retroperitonealer oder intrathorakaler Strukturen auftreten.

Die Hauptkomplikation der Vertebround Kyphoplastie besteht in der Zementfehllage mit Austritt von Zement in den Zwischenwirbelraum, in paravertebrale Venen oder den Spinalkanal. Obwohl die Zementleckagen häufig vorkommen, bleiben sie in der Mehrzahl asymptomatisch und damit ohne klinische Relevanz. Die Bedeutung der intradiskalen Zementlage ist bisher nicht hinreichend in Studien untersucht (Abb. 5).

Der Übertritt von Zement in den Pedikel verursacht in der Regel keine Beschwerden und bedarf keiner Korrektur. Das Herausragen eines Zementrests aus dem Pedikel in die Weichteile sollte jedoch vermieden werden, hier wird zur Infektionsvermeidung eine Bergung empfohlen.

Eine Zementleckage in den Spinalkanal kann zu einer Beengung des Myelons führen und bedarf ggf. bei Auftreten einer sofortigen Entlastung, um bleibende neurologische Störungen zu vermeiden (dorsale Clearance/Laminektomie). Hierbei wird eine zusätzliche dorsale Stabilisierung notwendig.

Gelegentlich treten bei beiden Verfahren passagere neurologische Störungen durch die Wärmeentwicklung bei der Polymerisierung auf. Auch das Vorkommen von Lungenembolien durch den Abfluss des Zements über lokale Venen wird vereinzelt in der Literatur beschrieben [26]. Daneben werden Fettembolien bei beiden Methoden erwähnt.

Neuere Untersuchungen zeigen eine geringere Komplikationsrate der Kyphoplastie gegenüber der Vertebroplastie bezüglich der unerwünschten Zementübertritte. Die Ursache hierfür ist die Verwendung eines dickflüssigeren Zements, welcher mit geringem Druck in einen durch den Ballon ausgeformten Hohlraum eingespritzt wird. Demgegenüber muss bei der Vertebroplastie ein dünnflüssigerer Zement mit höherem Druck appliziert werden.

Blutungen sind wie bei allen Eingriffen auch bei der Kypho- und Vertebroplastie möglich und ebenso ernst zu nehmen wie bei anderen Operationsverfahren. Fehlpunktionen können hier zu epiduralen oder subduralen Hämatomen führen, die gegebenenfalls zu entlasten sind.

Als seltene Komplikation werden allergische Reaktionen auf das verwendete Material beschrieben.

Die Vertebro- und Kyphoplastie scheinen einfache Verfahren. Bei der Indikationsstellung und der Ausführung sind die Komplikationsmöglichkeiten allerdings sehr hoch. 


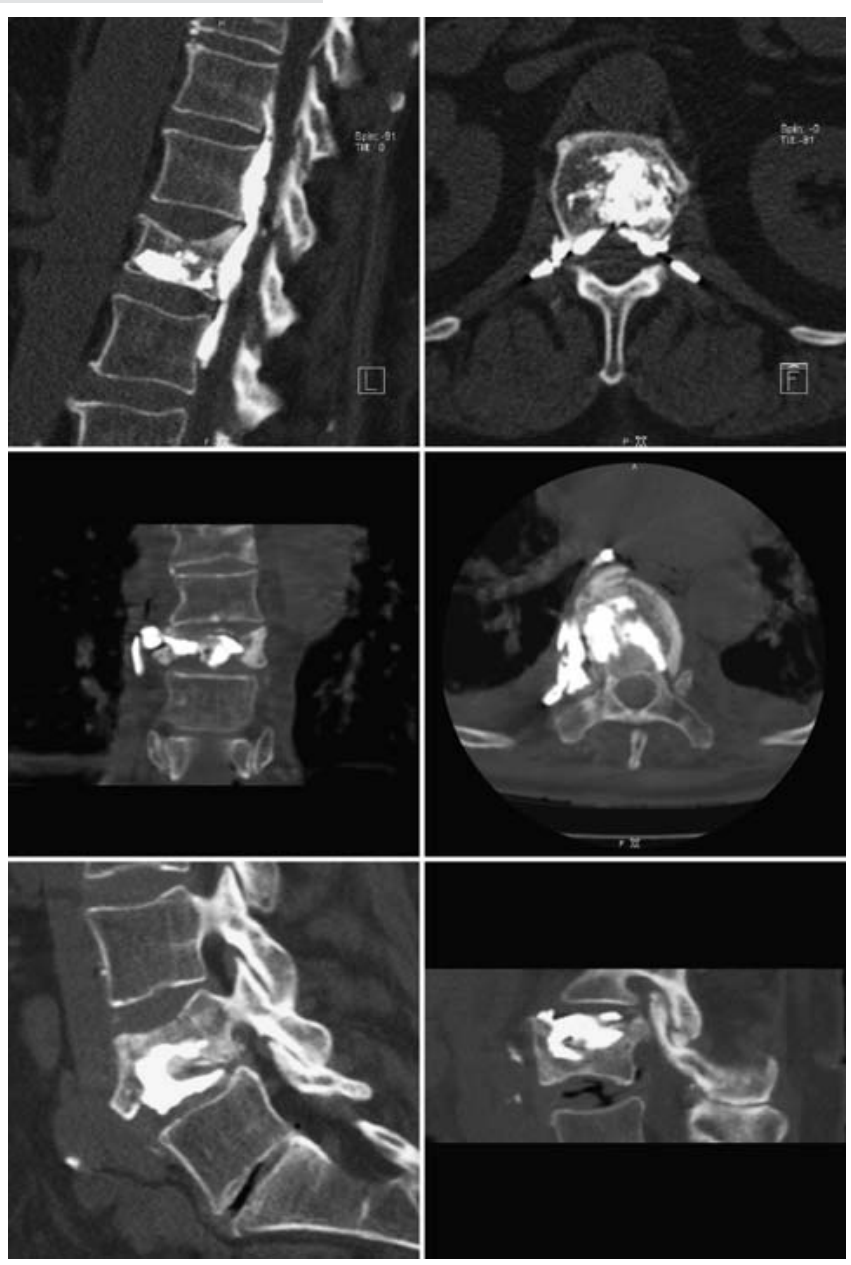

Abb. 5 Zementfehllage nach Kypho- und Vertebroplastie.

\section{Komplikationsmanagement bei postoperativen Problemen}

\section{Allgemeine Komplikationen}

Durch den Einsatz der Thromboseprophylaxe und vor allem die frühzeitige Mobilisierung durch zeitnahe, belastungsstabile Osteosynthesetechniken an der Wirbelsäule hat die Gefahr thrombembolischer Komplikationen deutlich abgenommen. Gefährdet sind allerdings Patienten mit neurologischen Komplikationen nach dem Trauma. Neben der nicht gegebenen Mobilisation kann es durch neurologische Störungen der Gefäßmuskulatur zu einer kritischen Verlangsamung des Blutflusses in den unteren Extremitäten kommen. Solche Patienten haben als Hochrisikopatienten zu gelten. Auch steigt bei diesen Verletzten das Dekubitusrisiko. Die eingeschränkten Mobilisationsmöglichkeiten auch im Liegen und oftmals auch posttraumatische Depressionen mit Antriebsarmut sorgen für einen hohen Pflegeaufwand mit regelmäßigen Lagewechseln.

\section{Psychische Veränderungen}

Patienten mit Wirbelsäulenverletzungen haben sich, auch wenn keine neurologischen Störungen vorliegen, das „Kreuz gebrochen“. „Ich werde nie wieder so leben und arbeiten können wie früher." Neben den oftmals kompensierbaren objektiven Unfallfolgen liegt der Anteil der Patienten mit posttraumatischen Belastungsstörungen in keiner Verletzungsgruppe so hoch wie bei Wirbelsäulenverletzten. Eine frühzeitige, idealerweise sofort postoperativ einsetzende psychologische Begleitbetreuung von Patient und Angehörigen kann einer solchen die Teilhabe erheblich einschränkenden Störung vorbeugen.

Eine besondere psychische Belastung stellt aufgrund der Stigmatisierung die Behandlung von Verletzungen der Halswirbelsäule mit dem Halo-Fixateur dar.

Allgemeine und psychische Probleme zu erkennen stellt hohe Anforderungen an die Krankenbeobachtung.

\section{Hämatome}

Postoperative Hämatome treten mit einer Häufigkeit von bis zu $20 \%$ auf. In der Regel handelt es sich um kleine oder flächige Blutansammlungen, die sich spontan resorbieren und eine problemlose Wundheilung nicht stören. Als Ursache gelten unzureichende Blutstillung am Ende der Operation oder auch unsachgemäßer Umgang mit den Weichteilen. Bedenken muss man aber, dass bei der Verwendung minimalinvasiver Techniken in den nicht einsehbaren Bereichen Gefäßläsionen nicht erkannt werden und so Hämatome entstehen können.

Ein zunehmendes Problem stellen Patienten unter gerinnungshemmender Medikation dar, bei denen eine Normalisierung der Gerinnungswerte präoperativ nicht abgewartet werden kann. Auch bei scheinbar gesunden jungen Menschen kann es zu medikamenteninduzierten Störungen der Thrombozytenaggregation kommen, hier muss eine genaue Anamnese erhoben werden („ASS ist nicht nur gegen Kopf-, sondern auch gegen Gliederschmerzen!“).

Hämatome sind oft der Beginn einer Infektion. Regelmäßige sonografische Kontrollen des Wundgebiets stellen die zuverlässigste Methode zur Erkennung auch tief im Implantatlager angesammelter Hämatome dar. Ab welcher Menge Hämatome operativ ausgeräumt werden sollen, ist der Erfahrung des Operateurs überlassen. Im Bereich der Hüfte werden Mengen zwischen 20 und $50 \mathrm{ml}$ als Grenze diskutiert, an der Wirbelsäule sind die anatomischen Beziehungen sehr viel enger, eine Revision also deutlich früher indiziert.

Auch im Bereich der Entnahme eines Knochenspans oder von Spongiosa am Beckenkamm treten häufig Hämatome auf. Sorgfältige Blutstillung auch mit Einlage von gerinnungshemmenden Substanzen helfen, diese Komplikation und eine sich möglicherweise daraus entwickelnde Infektion zu vermeiden. Die intraoperative Lokalanästhesie kann außerdem die oft nach Beckenknochenentnahme bestehende postoperative Schmerzsymptomatik des Patienten reduzieren.

Hämatome muss man aktiv suchen und frühzeitig revidieren.

Als Sonderform des Hämatoms ist der Hämatothorax nach ventraler Fusion 


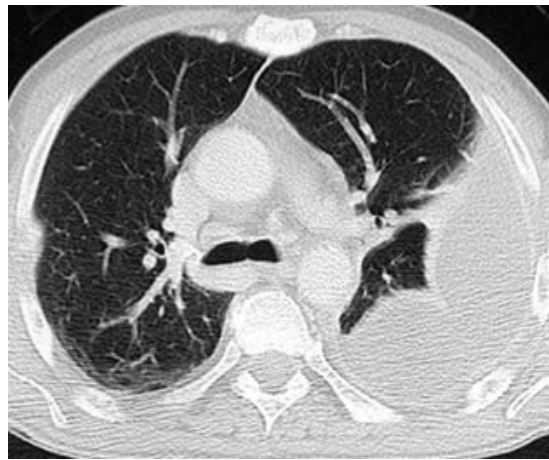

Abb. 6 Postoperativer Hämatothorax nach ventraler Fusion mit Wirbelkörperersatz und ventraler winkelstabiler Platte.

oder auch direkt posttraumatisch zu sehen. Eine Behandlung erfolgt durch Drainagen, in wenigen Ausnahmefällen ist eine Rethorakotomie mit Hämatomausräumung angezeigt. Problematisch sind solche Blutansammlungen in der Brusthöhle durch die Verdrängung des Lungengewebes und der daraus resultierenden Verminderung der Atemkapazität mit all ihren negativen Folgen (Abb.6) [14, 29,32].

\section{Infektionen}

In der Literatur wird die Häufigkeit postoperativer Infektionen mit 3-12\% angegeben. Da es sich bei Wirbelsäulenverletzungen in der Regel um geschlossene Frakturen handelt, ist die Inzidenz der postoperativen Infektkomplikationen eher im unteren Prozentbereich zu sehen. Die klinische Beobachtung, Laborverlaufskontrollen und vor allen die sonografischen Beobachtungen dienen der Früherkennung der Infektionen. Trotz der wie erwähnt engen anatomischen Verhältnisse ist die autochthone Rückenmuskulatur dick genug, um den Beginn tiefer Infekte zu verschleiern. Die sofortige operative Revision ist indiziert, konservative Versuche mit Antibiose bleiben immer ohne Erfolg.

Das operationstaktische Vorgehen ist abhängig von der Ausdehnung der Infekte und auch von der Erfahrung des Operateurs. Bei subkutanen Problemen, beruhend auf Fettgewebsnekrosen, können eine Revision mit Jetlavage, Einlegen eines VAC-Systems und der geplante Second Look zum Erfolg führen. Ist das Implantat allerdings in den Prozess mit einbezogen, muss eine Explantation des Fixateur-interne-Systems erfolgen, der Wechsel sollte dann spätestens auf ein Titanimplantat erfolgen, da bekanntlich die Adhäsion von Bakterien und die Biofilmbildung auf Titan wesentlich geringer ist als auf Stahl (Abb. 7).

Bei ausgedehnten infektiösen Prozessen verbietet sich ein einzeitiger Wechsel des Osteosynthesesystems; nach der Entfernung des Materials muss der Infekt zuerst saniert werden, um dann erneut eine operative Stabilisierung der Fraktur zu erreichen. In Ausnahmefällen kann, wie an den Extremitäten auch, der Fixateur externe eine hilfreiche Alternative sein, um den Patienten weiter zu mobilisieren. Das muss allerdings mit dem Patienten vorher eingehend besprochen werden; der Tragekomfort des Fixateur externe an der Wirbelsäule ist äußerst gering und hindert beim Liegen und beim Sitzen (Abb. 8).

Infekte nach ventraler Stabilisierung der Wirbelsäule sind äußerst selten und auch in der Literatur in sehr wenigen Einzelfällen beschrieben. Hier kann ein Behandlungsvorschlag nicht gemacht werden, die Therapieoptionen sind individuell zu wählen.

\section{Implantatassoziierte Komplikationen}

Unter implantatassoziierten Komplikationen versteht man Implantatfehllagen, Implantatversagen und Implantatlockerungen. Die Fehllagen wurden bereits als intraoperative Probleme behandelt.

Die Grundlage eines Implantatversagens, in der Regel ein Implantatbruch, sind biomechanische Probleme bei der Anwendung, welche $\mathrm{zu}$ einer Dauerschwingbelastung führen und damit zum Bruch. Bei der Osteosynthese der Wirbelsäule werden in der Regel bewegliche Segmente, die Junghans'schen Bewegungssegmente, mit dem Implantat überbrückt. Werden diese Segmente nicht ventral durch eine Spondylodese dauerhaft aufgegeben, kommt es bei jedem Schritt zu einer Schwingbelastung des Implantats. Biomechanische Studien haben gezeigt, dass hochstabile Fraktursysteme etwa eine Million Lastwechsel vertragen und dann versagen. Bei diesen Systemen brechen in der Regel die Pedikelschrauben am Eintrittspunkt in den Knochen und können nur mit erhöhtem Aufwand und dem Risiko von Komplikationen wie z.B. iatrogener Bogenbrüche entfernt werden. Sieht man dieses Problem präoperativ in der Bildgebung, sollte der Patient auf das Belassen des Restimplantats aufgeklärt werden.

Bei polyaxialen Systemen kommt es seltener zu Schraubenbrüchen, hier versagen in der Regel die Winkelstabilisierungen und es kommt zum ventralen Korrekturverlust. Der Bruch der Längsträger kann bei langstreckigen Versorgungen auftreten. Diese Probleme sind

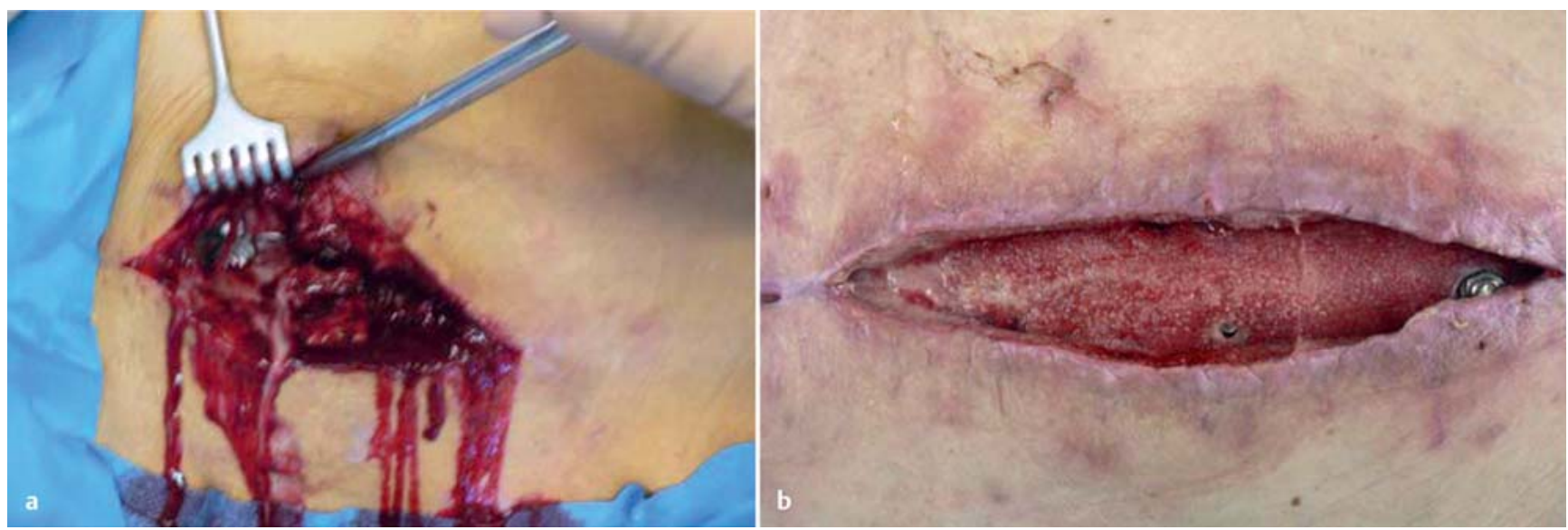

Abb. 7 a und b Akuter (a) und chronischer (b) Wundinfekt, ein Implantatwechsel ist zwingend notwendig. 


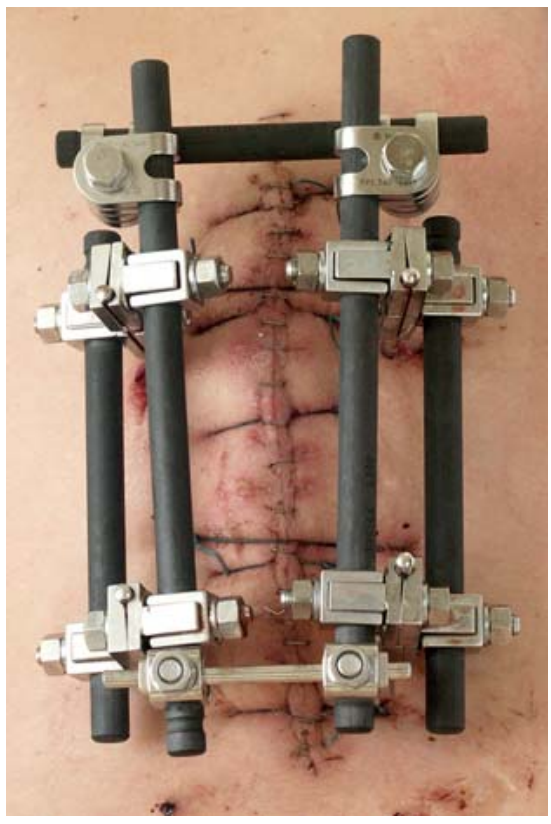

Abb. 8 Fixateur externe, eine seltene Rückzugsmöglichkeit bei postoperativen Infekten nach dorsaler Versorgung.

fast ausschließlich in der Spätphase einer Behandlung zu erwarten und können, da meist die Frakturen knöchern konsolidiert sind, durch eine ersatzlose Materialentfernung, gelegentlich unter Belassen von Restimplantat, behoben werden.

Ein größeres Problem stellt das Auslockern der Pedikelschrauben im Knochen dar. Gerade bei verminderter Knochenqualität, z.B. bei älteren Patienten, kommt es zu großen Substanzverlusten, die eine Revision in gleicher Höhe unmöglich werden lassen. Hier muss die Segmenthöhe verändert werden, was allerdings wieder zu einer Verlängerung der Schwingstrecke des Implantats führt. Zurzeit werden kanülierte Pedikelschrauben im Markt eingeführt, durch die Knochenzement zur Verstärkung des Schraubenlagers in den Wirbelkörper eingebracht werden kann. Ob und wie sich solche Implantate bewähren, muss beobachtet werden (Abb.9).

Gelegentlich findet man auch an der Wirbelsäule ein Cut-off der Pedikelschrauben bei verminderter Knochenqualität in den benachbarten Bandscheibenraum. Eine Entfernung des Implantats und bedarfsweiser Neuplatzierung ist dann notwendig (Abb. 10).

Seltener sind Probleme mit ventralen Implantaten zu beobachten. Eingebrachte Wirbelkörperersatzimplantate kön-

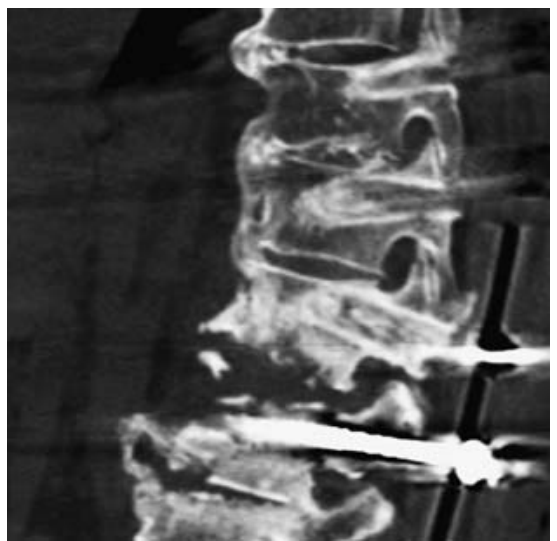

Abb.9 Große Knochensubstanzverluste nach Auslockern eines Fixateur interne bei verminderter Knochenqualität.

nen dislozieren $[17,33]$. Um eine Kompromittierung umliegender Strukturen mit durchaus tödlichen Folgen zu verhindern, ist eine Revision mit Neuplatzierung angezeigt $[5,11,20,21]$.

Auslockerungen der Stifte eines HaloFixateurs sind häufig und können durch regelmäßige Kontrollen und Nachziehen der Pins reduziert werden. Kommt es zu einer vollständigen Lockerung, muss eine Umsetzung der Pins oder ein Verfahrenswechsel erwogen werden.

Betrachtet man ventral eingebrachte kortikospongiöse Späne als Implantat, stellen Spanresorption und Pseudarthrosen der Anschlussstellen Implantatprobleme dar. Während eine Spanresorption immer zu einem Korrekturverlust führt und so zu einer Reoperation zwingt, sind die Pseudarthrosen durch Verwendung zusätzlicher ventraler Stabilisierungssysteme deutlich seltener geworden und stellen in der Regel kein revisionspflichtiges Problem mehr dar (Abb. 11) [34].

Implantatassoziierte Komplikationen resultieren in der Regel durch Nichtbeachtung der Biomechanik und der Knochenqualität.

\section{Neurologische Komplikationen}

Treten in der postoperativen Phase neurologische Ausfälle auf, stellt sich zuallererst die Frage, ob es sich nicht um übersehene, bereits präoperativ bestehende Störungen handeln kann. Dies ist in erster Linie dann zu erwarten, wenn der Patient im Rahmen der Erstversorgung intubiert werden musste und nicht einer subtilen präoperativen körperlichen Untersuchung unterzogen werden

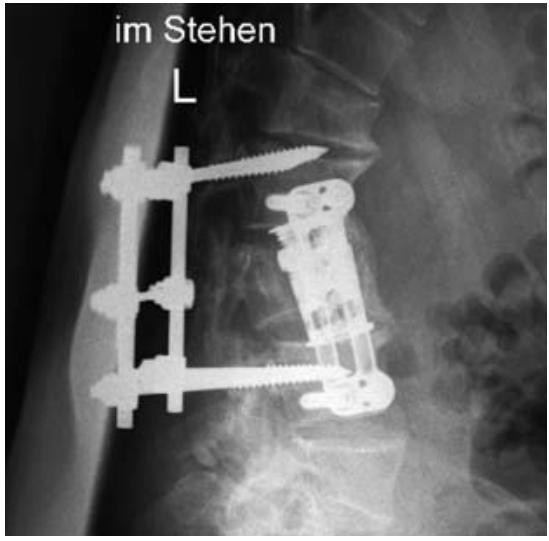

Abb. 10 Cut-off der Pedikelschrauben nach dorsoventraler Versorgung.

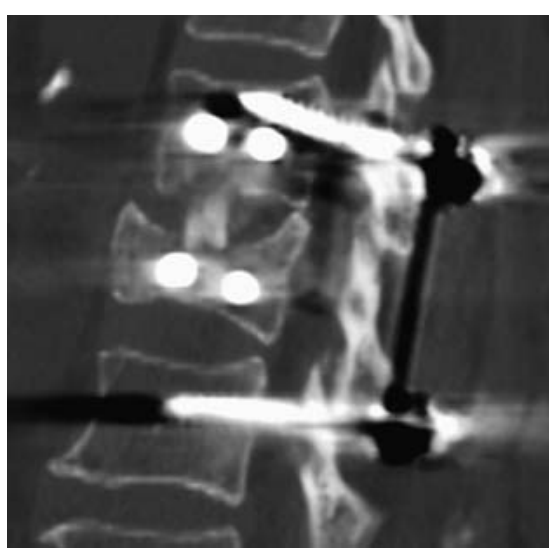

Abb. 11 Nicht vollständig eingeheilter Span mit zusätzlicher ventraler Stabilisierung durch ein winkelstabiles Implantat, in der Regel klinisch unbedeutend.

konnte $[9,18]$. Trotzdem muss dem Befund nachgegangen werden, eine CTKontrolle des operativen Ergebnisses ist unerlässlich. Bei dem geringsten Verdacht auf eine operative Fehlleistung, z.B. übersehene intraspinale Fragmente, Pedikelschraubenfehllagen oder auch intraspinale Hämatome muss eine unverzügliche operative Revision des Befunds erfolgen. In diesem Rahmen sei zur Diskussion gestellt, ob nicht bei präoperativ unklaren Verhältnissen ausschließlich Titanimplantate verwendet werden sollten, um auch postoperativ MRT-Untersuchungen zu ermöglichen.

\section{Seltene Komplikationen}

Bei der Durchsicht der Literatur stößt man gelegentlich auf Fallbeschreibungen mit seltenen Ereignissen. So sind zum Beispiel bei der Verwendung des HaloFixateurs bei der unbeabsichtigten Perforation der Tabula interna des Schädels Pininfektionen mit Auftreten von epidu- 


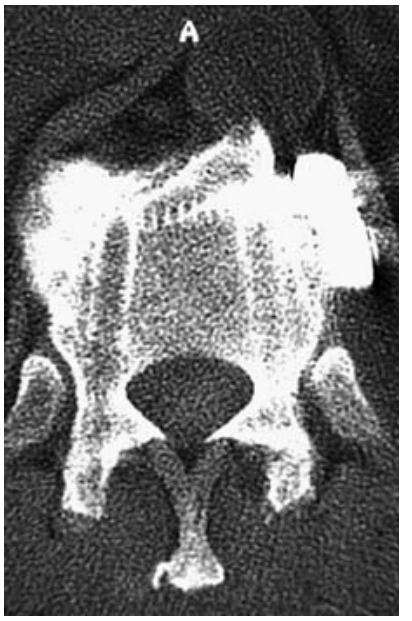

Abb. 12 Ein nicht korrekt eingebrachter Span arrodiert mit seiner Kante die Aorta.

ralen Abszessen beschrieben [25,28]. Bei ventraler Versorgung im Bereich der unteren Halswirbelsäule können durch die Nähe zur ventral verlaufenden Speiseröhre Schluckstörungen auftreten. Diese können im Verlauf rückläufig sein, es sind aber auch sekundäre Perforationen der Speiseröhre bei vorstehenden Implantaten beschrieben. Eine postoperative bestehende Heiserkeit bedarf der Abklärung zum Ausschluss einer Rekurrensparese. Bei erhaltener Kontinuität des N. recurrens ist mit einem Rückgang der Beschwerden zu rechnen.

Auch lumbale oder thorakale Gefäßläsionen, ob nun im Zusammenhang mit dem Trauma durch Distension oder im Zusammenhang mit der Versorgung durch Implantatfehllagen, werden beschrieben (Abb. 12) [31].

Solche seltenen Ereignisse bedürfen individueller Behandlungen.

\section{Langzeitprobleme der Vertebro- und Kyphoplastie}

Die hauptsächlichen Probleme dieser Verfahren im postoperativen Verlauf sind auf indikatorische Fehlleistungen zurückzuführen. Wenn die Pathologie des diskoligamentären Bewegungssegments missachtet wird und eine nicht klassifikationsgerechte Indikation zu diesem Verfahren führt, kommt es zwangsläufig zu Instabilitäten der Bewegungssegmente mit neurologischen Störungen und Schmerzkomplikationen [12]. Aufwendige sekundäre Stabilisationsverfahren mit Entfernung des Zements und dorsoventraler Versorgung sind die Folgen (Abb. 13).
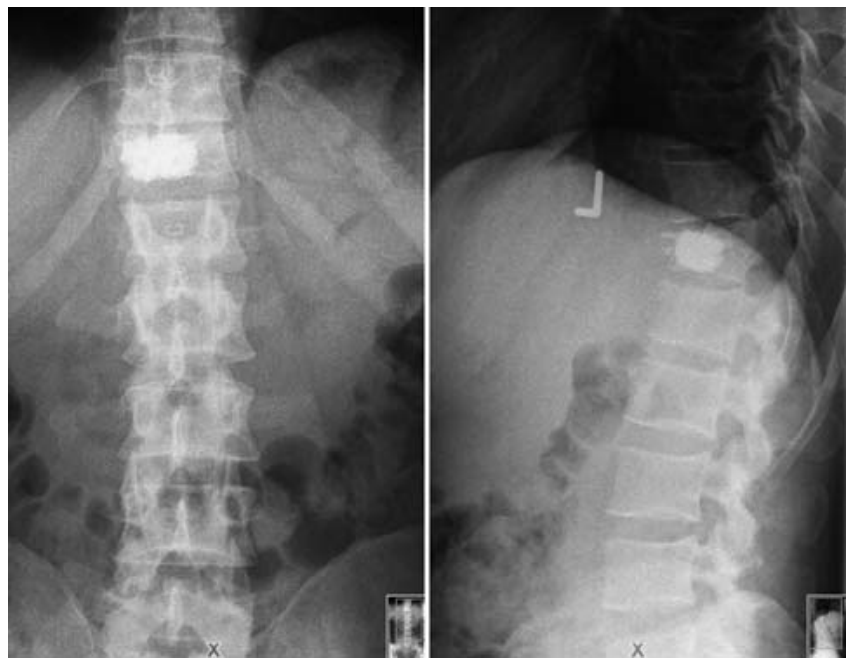

Abb. 13 Nicht indikationsgerecht ausgeführte operative Behandlung einer instabilen Verletzung mit Ballonkyphoplastie bei einem jungen Mann.
Bei der Versorgung von Sinterungen kann es zu Anschlusssinterungen durch die unterschiedlichen Elastizitätsmodule der versorgten und der unversorgten Wirbelkörper kommen. Wie und unter welchen Bedingungen diese entstehen, ist zurzeit Gegenstand verschiedener Untersuchungen. Bisher werden die sekundär gesinterten Wirbelkörper ebenfalls mit Zement stabilisiert.

Die Folgen eines nicht vollständig wieder aufgerichteten Wirbelkörpers und der daraus resultierenden Störung des Alignments der Wirbelsäule werden in der Literatur diskutiert, abschließende Ergebnisse liegen hier allerdings noch nicht vor.

\section{Literatur}

${ }^{1}$ Aydinli U, Karaeminogullari O, Tiskaya K et al. Dural tears in lumbar burst fractures with greenstick lamina fractures. Spine 2001; 26: E410-E415

2 Burdi M, Bono CM, Kauffman CP et al. Delayed diagnosis of a flexion-distraction (seat belt) injury in a patient with multiple abdominal injuries: a case report. Am J Orthop 2008; 37: 44-46

3 Campbell A, Yen D. Late neurologic deterioration after nonoperative treatment of a Chance fracture in an adolescent. Can J Surg 2003; 46: 383-385

${ }^{4}$ Carl AL, Matsumoto M, Whalen JT. Anterior dural laceration caused by thoracolumbar and lumbar burst fractures. J Spinal Disord 2000; 13: 399-403

${ }^{5}$ Chen HC, Tzaan WC, Chen TY et al. Esophageal perforation complicating with spinal epidural abscess, iatrogenic or secondary to first thoracic spine fracture? Acta Neurochir (Wien) 2005; 147: 431-434

${ }^{6}$ Cho SK, Lenke LG, Hanson D. Traumatic noncontiguous double fracture-dislocation of the lumbosacral spine. Spine J 2006; 6: 534-538

7 Chou D, Hartl R, Sonntag VK. Conus medullaris syndrome without lower-extremity involve- ment in L-1 burst fractures: report of four cases. J Neurosurg Spine 2006; 4: 265-269

8 Cokluk C, Aydin K. Segmental artery pseudoaneurysm associated with thoracic spinal fracture. Turk Neurosurg 2007; 17: 142-146

${ }^{9}$ Dai LY, Wang XY, Jiang LS. Neurologic recovery from thoracolumbar burst fractures: is it predicted by the amount of initial canal encroachment and kyphotic deformity? Surg Neurol 2007; 67: 232-237

${ }^{10}$ Dalvie SS, Burwell M, Noordeen MH. Haemothorax and thoracic spinal fracture. A case for early stabilization. Injury 2000; 31: 269270

11 Domenicucci M, Ramieri A, Lenzi J et al. Pseudo-aneurysm of a lumbar artery after flexion-distraction injury of the thoraco-lumbar spine and surgical realignment: rupture treated by endovascular embolization. Spine 2008; 33: E81-E84

12 Evans AJ, Kip KE, Boutin SM. Development and psychometric properties of the vertebral compression fracture pain and functional disability questionnaire. J Neurosurg Spine 2006; 5: 217-223

${ }^{13}$ Fayyazi AH, Segal L. Surgical excision of symptomatic lumbar spinous process pseudoarthrosis. J Spinal Disord Tech 2004; 17: 439441

14 Frangen TM, Ruppert S, Muhr G et al. [Respiratory failure in thoracic spine injuries. Does the timing of dorsal stabilization have any effect on the clinical course in multiply injured patients?]. Orthopade 2007; 36: 365-371

15 Hessler C, Raimund F, Regelsberger $J$ et al. Komplikationen bei operativer Dekompression an der tumorinfiltrierten Wirbelsäule. Chirurg 2007; 78: 915-927

${ }^{16}$ Hiraizumi Y. Spinal cord anterior decompression for delayed spinal cord paralysis after osteoporotic vertebral compression fracture: application of thoracoscopic approach. Chir Narzadow Ruchu Ortop Pol 2008; 73: 67-73

17 Johnson MG, Fisher CG, Boyd $M$ et al. The radiographic failure of single segment anterior cervical plate fixation in traumatic cervical flexion distraction injuries. Spine 2004; 29: 2815-2820

${ }^{18} \mathrm{Kim}$ HS, Kim NH, Lee HM et al. Sexual dysfunction in men with paraparesis in lumbar burst fractures. Spine 2000; 25: 2187-2190

${ }^{19}$ Kirkpatrick AW, McKevitt E. Thoracolumbar spine fractures: is there a problem? Can J Surg 2002; 45: 21-24 
${ }^{20}$ Langen HP, Kühling J, Grüber C et al. Late erosion of the aorta after dorsal-ventral care of a L1 fracture with prominent chip position. Eur J Trauma Emerg Surg 2008; 34: 302-304

21 Lifshutz J, Lidar Z, Maiman D. Thoracic aortic pseudoaneurysm after spine trauma in ankylosing spondylitis. Case report. J Neurosurg Spine 2005; 2: 218-221

22 Masala S, Fiori R, Massari F et al. Kyphoplasty: indications, contraindications and technique. Radiol Med 2005; 110: 97-105

${ }^{23}$ Mehta JS, Reed MR, McVie JL et al. Weightbearing radiographs in thoracolumbar fractures: do they influence management? Spine 2004; 29: 564-567

${ }^{24}$ O'Brien JR, Krushinski E, Zarro CM et al. Esophageal injury from thoracic pedicle screw placement in a polytrauma patient: a case report and literature review. J Orthop Trauma 2006; 20: 431-434

25 Papagelopoulos PJ, Sapkas GS, Kateros KT et al. Halo pin intracranial penetration and epidural abscess in a patient with a previous cranioplasty: case report and review of the literature. Spine 2001; 26: E463-E467

26 Pleser M, Roth R, Wörsdörfer $O$ et al. Lungenembolie durch PMMA bei perkutaner Vertebroplastie: Fallbericht und Literaturübersicht. Unfallchirurg 2004; 107: 807-811

27 Reinhold M, Schmid R, Knop C et al. Komplikationsspektrum operativ versorgter Wirbelsäulenverletzungen - Eine Analyse der Multicenterstudien I und II der AG Wirbelsäule. Trauma Berufskrankh 2005; 7 (Suppl. 2): S281-S291
${ }^{28}$ Rodriguez M, Tyberghien A, Matge G. Asymptomatic vertebral artery injury after acute cervical spine trauma. Acta Neurochir (Wien) 2001; 143: 939-945

29 Schinkel C, Greiner-Perth R, SchwienhorstPawlowsky $G$ et al. [Does timing of thoracic spine stabilization influence perioperative lung function after trauma?]. Orthopade 2006; 35: 331-336

30 Stich R, Makkas M. Fehler und Gefahren bei chirurgischen Operationen. Jena: Verlag von Gustav Fischer; 1923

31 Vaidyanathan S, Hughes P, Sett P et al. Injury to renal vein during fixation of fractured lumbar spine and subsequent atrophy of kidney in a female patient with spinal cord injury and paraplegia. Spinal Cord 2003; 41: 527-529

32 van Raaij TM, Slis HW, Hoogland PH et al. Massive haemothorax following thoracic vertebral fracture. Injury 2000; 31: 202-203

33 von Rahden BH, Stein HJ, Scherer MA. Late hypopharyngo-esophageal perforation after cervical spine surgery: proposal of a therapeutic strategy. Eur Spine J 2005; 107: 1152-1161

${ }^{34}$ Wälchli B, Heini P, Berlemann U. Korrekturverlust nach dorsaler Stabilisierung von Berstungsfrakturen des thorakolumbalen Überganges. Unfallchirurg 2001; 104: 742-747

35 Wick MC, Rieger M. Initially overseen vertebral body luxation: diagnosed by dynamic fluoroscopy due to delayed dysphagia. Eur Spine J 2007; 16 (Suppl. 3): S278-S282. Epub 2007 Aug 14

\section{Dr. med. Jan Baranczyk}

Oberarzt

Bereichsleiter Wirbelsäulenchirurgie

Klinik für Unfall-, Hand- und

Wiederherstellungschirurgie

Dr. med. Hans Peter Langen Oberarzt

Klinik für Unfall-, Hand- und

Wiederherstellungschirurgie

Ina Schmidt

Unfallforschung Berlin-Buch

Dr. med. Jörg Schmidt

Chefarzt

Klinik für Unfall-, Hand- und

Wiederherstellungschirurgie HELIOS Klinikum Berlin-Buch

Schwanebecker Chaussee 50 13125 Berlin

joerg.schmidt@helios-kliniken.de 